\title{
Actuator and Sensor Positioning Optimization in Control Design for a Large BWB Passenger Aircraft
}

\author{
A. Schirrer, C. Westermayer, M. Hemedi, and M. Kozek \\ Division of Control and Process Automation, Institute of Mechanics and Mechatronics, Vienna University of Technology, \\ Wiedner Hauptstraße 8 / E325/A5, 1040 Vienna, Austria
}

Correspondence should be addressed to A. Schirrer, alexander.schirrer@tuwien.ac.at

Received 31 January 2011; Accepted 17 March 2011

Academic Editors: M. Ahmadian, D. Kalempa, and F. Liu

Copyright @ 2011 A. Schirrer et al. This is an open access article distributed under the Creative Commons Attribution License, which permits unrestricted use, distribution, and reproduction in any medium, provided the original work is properly cited.

This paper states an approach to actuator and sensor positioning optimization and design in the control system design of a large blended wing body (BWB) passenger aircraft. Numerous objectives have to be achieved by the control system: loads alleviation, vibration attenuation, and the fulfillment of handling quality requirements. Exploiting the system structure and existing system knowledge (excitation, comfort, and load formulations), evaluation criteria are designed to assess actuator and sensor effectiveness and efficiency for the aircraft dynamic range of interest. The tasks of optimal actuator and sensor positioning, actuator sizing, and actuator bandwidth requirements are investigated, whereby solutions that are robust are sought with respect to parameter variations. The results are shown on a BWB passenger aircraft model and verified using a normalized closed-loop performance assessment approach.

\section{Introduction}

The high complexity of a large-scale system design procedure, such as the control system design of a passenger aircraft, is challenging. The modeling issues are manifold, also involving the interdependence of design decisions and the lack of perfect model information in the design process.

In this work, a part of control system design performed within the predesign stage of a large blended wing body (BWB) passenger aircraft (see Figure 1) is reported: the robust optimal selection of inputs and outputs for a vibration control system. Also the related evaluation and system design methods are proposed. This task is comprised of gathering and formulating control design objectives, defining the scope and methods of optimization, choosing appropriate design weights to incorporate excitations and performance objectives, and finally computing optimization results and interpreting them with respect to the system design tasks. The flexible aircraft model under study stems from the ACFA 2020 EU FP7 research project [1].

The special properties of flexible structure systems are described in detail in [2]. Fundamental properties of multiinput multi-output (MIMO) control systems and state space system calculus are presented in [3].
Many methods of control system input/output evaluation and selection exist nowadays, and an excellent survey is given by [4]. More recent sources are given by $[2,5$, $6]$. The evaluation criteria proposed in $[2,6]$ assess state controllability and observability in a weighted sense. They are combined into a recently proposed criterion to exploit their individual advantages [7].

Experimental results of actuator and sensor positioning optimization are given in $[6,8-10]$ for Piezo actuators and strain sensors. In actuator positioning, the task is typically to find those actuators out of a set of candidates which robustly maximize a controllability-related measure. Optimally positioned sensors can, in turn, be defined by their optimal capability to detect structural behavior. In this work, this is required in all relevant operating points of the aircraft. In this sense, a robust placement of actuators and sensors has to be obtained. This is even more important as the dynamic properties of the aircraft change strongly across the admissible parameter range. For the present study, a multimodel approach has been employed, using a set of models with two varying parameters: fuel load and payload.

The remainder of this work is organized as follows. First, the well-known modal modeling approach for flexible 


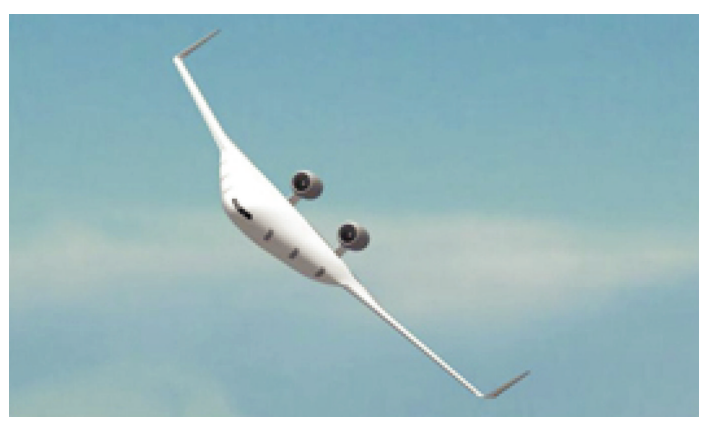

FIGURE 1: Large-scale blended wing body (BWB) passenger aircraft.

structures is outlined in Section 2. The positioning criteria and input/output design methodologies are presented and developed in Section 3. Then, the utilized aircraft model is presented in Section 4. Finally, the design tasks are carried out on the aircraft model and the results are collected in Section 5, concluded by final remarks and an outlook to future work.

\section{Structure Modeling}

2.1. Nodal Models. The dynamics of a linear-elastic, flexible structure system can be modeled as a state space dynamic system in terms of nodal coordinates (displacements $\mathbf{q}$ and velocities $\dot{\mathbf{q}}$ of structure nodes), for example, obtained by an FE formulation

$$
\mathbf{x}_{n}=\left[\begin{array}{c}
\mathbf{q}_{n} \\
\dot{\mathbf{q}}_{n}
\end{array}\right] .
$$

The state equation reads

$$
\dot{\mathbf{x}}_{n}=\underbrace{\left[\begin{array}{cc}
\mathbf{0}_{[n \times n]} & \mathbf{I}_{[n \times n]} \\
-\mathbf{M}^{-1} \mathbf{C} & -\mathbf{M}^{-1} \mathbf{K}
\end{array}\right]}_{\mathbf{A}_{n}} \mathbf{x}_{n}+\underbrace{\left[\begin{array}{c}
\mathbf{0}_{[n \times r]} \\
\mathbf{M}^{-1} \mathbf{B}
\end{array}\right]}_{\mathbf{B}_{n}} \mathbf{u},
$$

with $\mathbf{M}, \mathbf{C}$, and $\mathbf{K}$ as $(n \times n)$ mass-, damping-, and stiffness matrices, $\mathbf{B}$ as $(n \times r)$ input matrix, $\mathbf{0}$ and $\mathbf{I}$ as zero and identity matrices with the indicated dimensions. The system output can be written as

$$
\mathbf{y}=\underbrace{\left[\begin{array}{ll}
\mathbf{C}_{o q} & \mathbf{C}_{o v}
\end{array}\right]}_{\mathbf{C}_{n}} \mathbf{x}_{n},
$$

with $\mathbf{C}_{o q}$ and $\mathbf{C}_{o v}$ as $(s \times n)$ displacement and velocity output matrices, respectively.

2.2. Modal Form. For linear-dynamic systems with lowdamped oscillatory modes, choosing modal coordinates leads to a simple, decoupled model representation. Utilizing the similarity transformation $\mathbf{q}_{n}=\mathbf{T} \mathbf{q}_{m}$ (modal coordinates $\mathbf{q}_{m}$, eigenvector matrix $\mathbf{T}=\boldsymbol{\phi}=\left[\phi_{1} \cdots \phi_{n}\right]$ of the undamped eigenvector/eigenvalue problem $\mathbf{K} \phi_{i}=\omega_{i}^{2} \mathbf{M} \phi_{i}$, $i=1, \ldots, n)$ and choosing the numerically well-conditioned state vector

$$
\mathbf{x}_{m}=\left[\begin{array}{c}
\mathbf{x}_{m 1} \\
\vdots \\
\mathbf{x}_{m n}
\end{array}\right], \quad \mathbf{x}_{m i}=\left[\begin{array}{c}
\omega_{i} q_{m i} \\
\dot{q}_{m i}
\end{array}\right]
$$

yields the modal state space model

$$
\begin{gathered}
\dot{\mathbf{x}}_{m}=\mathbf{A}_{m} \mathbf{x}_{m}+\mathbf{B}_{m} \mathbf{u}, \\
\mathbf{y}=\mathbf{C}_{m} \mathbf{x}_{m},
\end{gathered}
$$

or, in transfer function notation,

$$
\mathbf{G}(s)=\mathbf{C}_{m}\left(s \mathbf{I}-\mathbf{A}_{m}\right)^{-1} \mathbf{B}_{m},
$$

with a $(2 \times 2)$ block-diagonal structure for each mode $i=$ $1, \ldots, n$

$$
\begin{gathered}
\mathbf{A}_{m i}=\left[\begin{array}{cc}
0 & \omega_{i} \\
-\omega_{i} & -2 \zeta_{i} \omega_{i}
\end{array}\right], \quad \mathbf{B}_{m i}=\left[\begin{array}{c}
\mathbf{0} \\
\mathbf{b}_{m i}^{T}
\end{array}\right], \\
\mathbf{C}_{m i}=\left[\begin{array}{ll}
\frac{\mathbf{c}_{m q i}}{\omega_{i}} & \mathbf{0}
\end{array}\right], \\
\mathbf{A}_{m}=\operatorname{diag}\left(\mathbf{A}_{m i}\right), \quad \mathbf{B}_{m}=\left[\begin{array}{c}
\mathbf{B}_{m 1} \\
\mathbf{B}_{m 2} \\
\vdots \\
\mathbf{B}_{m n}
\end{array}\right], \\
\mathbf{C}_{m}=\left[\begin{array}{llll}
\mathbf{C}_{m 1} & \mathbf{C}_{m 2} & \ldots & \mathbf{C}_{m n}
\end{array}\right] .
\end{gathered}
$$

The modal form, its advantages, and related methods are detailed in [2] and summarized in [7].

2.3. Acceleration Sensors. Given the displacement outputs for mode $i$

$$
\mathbf{y}=\left[\begin{array}{cc}
\frac{\mathbf{c}_{m q i}}{\omega_{i}} & 0
\end{array}\right]\left[\begin{array}{c}
\omega_{i} q_{m i} \\
\dot{q}_{m i}
\end{array}\right]=\mathbf{c}_{m q i} q_{m i},
$$

it is possible to formulate the corresponding acceleration outputs by eliminating $\ddot{q}_{\mathrm{m} i}$ via (5)

$$
\begin{aligned}
\ddot{\mathbf{y}} & =\mathbf{c}_{m q i} \ddot{q}_{m i} \\
& =\left[\begin{array}{ll}
-\mathbf{c}_{m q i} & -2 \zeta_{i} \omega_{i} \mathbf{c}_{m q i}
\end{array}\right]\left[\begin{array}{c}
\omega_{i} q_{m i} \\
\dot{q}_{m i}
\end{array}\right]+\mathbf{c}_{m q i} \mathbf{b}_{m i}^{T} \mathbf{u} .
\end{aligned}
$$

2.4. Linearization of Nonlinear Systems. Let a nonlinear system be described in a general state space formulation

$$
\begin{gathered}
\dot{\mathbf{x}}(t)=\mathbf{f}(\mathbf{x}(t), \mathbf{u}(t), \boldsymbol{\rho}(t)), \\
\mathbf{y}=\mathbf{g}(\mathbf{x}(t), \mathbf{u}(t), \boldsymbol{\rho}(t)),
\end{gathered}
$$


where $\mathbf{x}(t), \mathbf{u}(t)$, and $\boldsymbol{\rho}(t)$ are the state, input, and exogenous parameter vector signals, respectively. The linearization of (11) in an equilibrium point $\left(\mathbf{x}_{0}, \mathbf{u}_{0}, \boldsymbol{\rho}_{0}\right)$ with respect to $x$ and $u$ yields the linearized parameter-dependent state space system at fixed parameter values $\rho_{0}[11]$

$$
\begin{aligned}
& \Delta \dot{\mathbf{x}}(t)=\mathbf{A}\left(\rho_{0}\right) \Delta \mathbf{x}(t)+\mathbf{B}\left(\rho_{0}\right) \Delta \mathbf{u}(t), \\
& \Delta \mathbf{y}(t)=\mathbf{C}\left(\boldsymbol{\rho}_{0}\right) \Delta \mathbf{x}(t)+\mathbf{D}\left(\boldsymbol{\rho}_{0}\right) \Delta \mathbf{u}(t),
\end{aligned}
$$

in the deviation variables $\Delta \mathbf{x}(t)=\mathbf{x}(t)-\mathbf{x}_{0}, \Delta \mathbf{u}(t)=\mathbf{u}(t)-\mathbf{u}_{0}$, and $\Delta \mathbf{y}(t)=\mathbf{y}(t)-\mathbf{y}_{0}$. In the following, it is assumed that no feed-through term exists $(\mathbf{D}=0)$. Acceleration sensors (leading to nonzero D entries) can be incorporated by suitable transformations under the condition that the original system exhibits zero feed-through [2].

2.5. Incorporation of Design Constraints by System Weighting. A general, yet simple, solution to incorporating design constraints and conditions into system analysis is to apply a physically motivated input/output weighting to the system model before the actual system evaluation. Modal system representations allow simplified consideration of modewise weights if the weighting functions fulfil mild regularity conditions (see [2]).

Let $\left(\mathbf{A}_{m}, \mathbf{B}_{m}, \mathbf{C}_{m}\right)$ be a modal MIMO state-space system description (5). Consider a sufficiently smooth scalar transfer function $W_{\mathrm{o}}(\mathrm{j} \omega)$ for system output weighting (compare [2]). The output-weighted system $\left(\mathbf{A}_{m}, \mathbf{B}_{m}, \mathbf{C}_{m, w}\right)$ is obtained by modewise output scaling by the values $\left|W_{\mathrm{o}}\left(\mathrm{j} \omega_{i}\right)\right|$

$$
\mathbf{C}_{m, w}=\left[\begin{array}{lll}
\left|W_{o}\left(\mathrm{j} \omega_{1}\right)\right| \mathbf{C}_{m 1} & \cdots & \left|W_{o}\left(\mathrm{j} \omega_{n}\right)\right| \mathbf{C}_{m n}
\end{array}\right] .
$$

Analogously, frequency input weighting translates to modewise scaling of the input matrix (with weighting function $\left.W_{i}(\mathrm{j} \omega)\right)$

$$
\mathbf{B}_{m, w}=\left[\begin{array}{c}
\mathbf{B}_{m 1}\left|W_{i}\left(\mathrm{j} \omega_{1}\right)\right| \\
\mathbf{B}_{m 2}\left|W_{i}\left(\mathrm{j} \omega_{2}\right)\right| \\
\vdots \\
\mathbf{B}_{m n}\left|W_{i}\left(\mathrm{j} \omega_{n}\right)\right|
\end{array}\right] .
$$

Finally, weightings on actuators or sensors are usually applied by diagonal scaling matrices in series connection to the plant. The weighted system $\left(\mathbf{A}_{m}, \mathbf{B}_{m}, \mathbf{C}_{m, w}\right)$ has the same modal properties as the original system (natural frequencies, damping); only the mode gains are affected by the weighting process.

\section{Methodology}

This section develops a range of methods to study actuator and sensor positioning, actuator sizing, and actuator bandwidth estimation. First, an short discussion on input/output selection criteria is given. Then, algorithms based on two energy-based criteria for actuator and sensor positioning are outlined. A sensor positioning algorithm is defined and an LQG closed-loop validation procedure is proposed.
3.1. Overview. A broad overview on input/output selection methods is given by [4]. One widely applied approach is to maximize state controllability- or observability-related measures, or also energy transfer quantities.

Advantages are as follows.

(i) They provide qualitative statements over a wide range of control system architectures.

(ii) Physical interpretation is possible in the case of structure mechanics.

Deficits are as follows.

(i) Quantitative statements often depend on artificial weighting factors.

(ii) Robustness with respect to parameter variations is not addressed explicitly.

(iii) These methods might be suboptimal for certain controller architectures or design methods.

Alternatively, the positioning decision could exploit the future control design process by testing a given positioning decision, using a fully designed control system (e.g., $\mathscr{H}_{\infty}$ optimal robust controller designed by DK-iteration [3]) and its closed-loop behavior.

However, the following limitations are evident.

(i) This approach requires high computational and modeling effort.

(ii) Verification is difficult or not feasible until the final design has been carried out.

3.2. Energy-Based Positioning Criteria. Energy-based criteria and the closely related controllability-/observability-based criteria are widely applied (see [4]). Two existing methodologies for actuator and sensor positioning criteria are considered in the following: one is proposed by Gawronski [2], the other is proposed by the authors in [7].

3.2.1. Gawronski's Criteria. A mode-based aggregate index that quantifies total energy transfer into or out of the structure has been proposed by Gawronski in [2]. For a system in modal form (5)-(6) and the $j$ th actuator, the actuator index $\sigma_{\text {act }, j}$ is defined as

$$
\sigma_{\mathrm{act}, j}=\sqrt{\sum_{i=1}^{n} \sigma_{i j}{ }^{2}}, \quad \sigma_{i j}=w_{i j} \frac{\left\|\mathbf{G}_{i j}\right\|_{2}}{\|\mathbf{G}\|_{2}},
$$

where

$$
\left\|\mathbf{G}_{i j}(\mathbf{j} \omega)\right\|_{2} \cong \frac{\left\|\mathbf{B}_{m i j}\right\|_{2}\left\|\mathbf{C}_{m i}\right\|_{2}}{2 \sqrt{\zeta_{i} \omega_{i}}}
$$

is the $\mathscr{H}_{2}$ norm of the $i$ th mode and $j$ th actuator, $\|\mathbf{G}\|_{2}$ is the $\mathscr{H}_{2}$ norm of the system, $\zeta_{i}$ and $\omega_{i}$ are the modal damping and frequency of mode $i$, and $w_{i j}$ is a weighting factor for mode and actuator weighting. 
Analogously, sensor placement indices are defined by using the quantities related to the $k$ th sensor

$$
\sigma_{\text {sens }, k}=\sqrt{\sum_{i=1}^{n} \sigma_{i k}{ }^{2}}, \quad \sigma_{i k}=w_{i k} \frac{\left\|\mathbf{G}_{i k}\right\|_{2}}{\|\mathbf{G}\|_{2}},
$$

where

$$
\left\|\mathbf{G}_{i k}(\mathrm{j} \omega)\right\|_{2} \cong \frac{\left\|\mathbf{B}_{m i}\right\|_{2}\left\|\mathbf{C}_{m i k}\right\|_{2}}{2 \sqrt{\zeta_{i} \omega_{i}}} .
$$

This aggregating criterion does not prevent the placement of actuators or sensors at nodes of higher-order modes.

3.2.2. Proposed Combined Criterion. An extended positioning criterion has been proposed by the authors in [7] that combines features of criteria proposed in $[2,6,12]$. It adds the requirement that a set of modes be controllable or observable to result in a nonzero criterion value. For actuator placement the newly proposed performance index is defined as

$$
\pi_{\mathrm{act}, j}=\sqrt{\sum_{i=1}^{n}\left(\frac{\left\|\mathbf{B}_{m i j}\right\|_{2}\left\|\mathbf{C}_{m i}\right\|_{2}}{2 \sqrt{\zeta_{i} \omega_{i}}}\right)^{2}} \cdot \sqrt[2 n]{\operatorname{det}\left(\mathbf{W}_{\mathrm{c} j}\right)},
$$

with $\mathbf{B}_{m i j}$ as the input matrix for the $j$ th actuator under study, $\mathbf{C}_{m i}$ as the performance output matrix, and $n$ as the number of modeled modes. The controllability Gramian $\mathbf{W}_{\mathrm{c} j}$ is computed for the system with single input $j$ and the set of modes that are required to be controllable. Note that this requirement prevents placement in and near modal nodes which improves robustness against small changes of the modal node locations.

For sensor placement the performance index is defined as

$$
\pi_{\mathrm{sens}, k}=\sqrt{\sum_{i=1}^{n}\left(\frac{\left\|\mathbf{B}_{m i}\right\|_{2}\left\|\mathbf{C}_{m i k}\right\|_{2}}{2 \sqrt{\zeta_{i} \omega_{i}}}\right)^{2}} \cdot \sqrt[2 n]{\operatorname{det}\left(\mathbf{W}_{\mathrm{o} k}\right)},
$$

with $\mathbf{B}_{m i}$ as the disturbance input matrix, $\mathbf{C}_{m i k}$ as the output matrix for the $k$ th sensor under study, and $\mathbf{W}_{\mathrm{o} k}$ as the observability Gramian of the system with single output $k$ and the modes required to be observable.

3.3. Avoiding Sensor Clustering. When selecting several sensors out of a large candidate set based on the performance indices in (20), nearly-optimal and closely positioned sensors tend to be chosen. However, they typically yield little additional information on the modes. One countermeasure proposed in [2] is to employ a simple correlation distance

$$
r_{k l}=\frac{\mathbf{g}_{k}^{T} \mathbf{g}_{l}}{\left\|\mathbf{g}_{k}\right\|\left\|\mathbf{g}_{l}\right\|},
$$

where $\mathbf{g}_{k}=\left[\left\|\mathbf{G}_{1 k}\right\|_{2}^{2} \cdots\left\|\mathbf{G}_{n k}\right\|_{2}^{2}\right]^{T}$ is the vector of squared mode gains of the excitation-sensor transfer function $\mathbf{G}_{i k}$ (mode $i$, sensor $k$ ). The quantity $r_{k l}$ equals 1 for perfectly correlated (redundant) sensors $k$ and $l$, and 0 if they are orthogonal in terms of mode gains. By choosing to include a sensor only when it is sufficiently uncorrelated, the clustering of correlated sensors can be avoided.

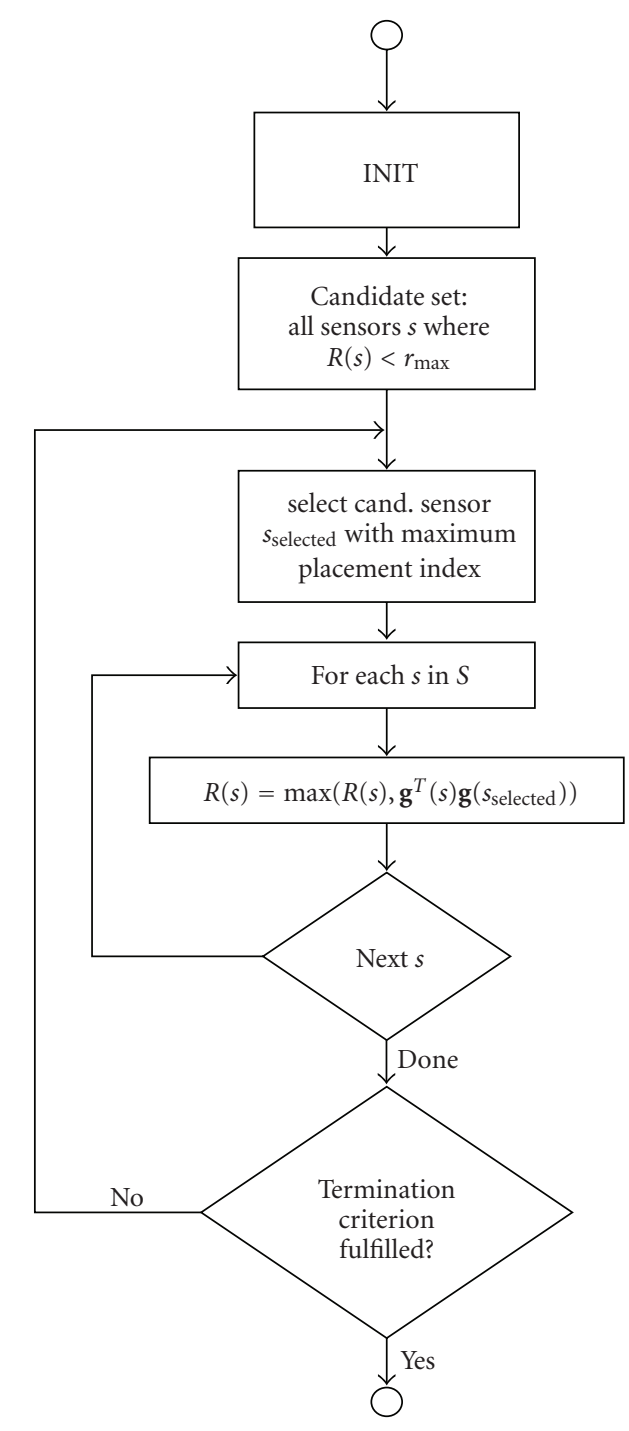

FIGURE 2: Flowchart of sensor positioning algorithm.

3.4. Sensor Positioning Algorithm. Figure 2 depicts the proposed sensor positioning algorithm. The best sensors (collected in set $S$ ) according to the used positioning index ((17) or (20)) are selected and this set is reduced iteratively by computing the updated worst-case mode gain correlation $R(s)$ (due to the max expression) and excluding all sensors that show a correlation index higher than the specified correlation threshold $r_{\max }$. This is repeated until a termination criterion is fulfilled, such as reaching a desired number of selected sensors or the event that all remaining sensors yield performance index values below a required threshold.

\subsection{Validation by Normalized LQG Closed-Loop Comparison}

3.5.1. Validation Procedure. A normalized LQG closed-loop validation (detailed in [13]) is used to support the statements of the developed methods. The idea is to design a normalized LQG controller (here, in the sense of fixed controller $\mathscr{H}_{2}$ norm, see Section 3.5.2) for each actuator positioning 
configuration and to evaluate the closed-loop performance. This is shown as a flow chart in Figure 3 and has to be repeated for each actuator.

In the LQ design, the quadratic cost function

$$
J=\int\left(\mathbf{x}^{T} \mathbf{Q} \mathbf{x}+\mathbf{u}^{T} \mathbf{R} \mathbf{u}\right) d t \longrightarrow \min
$$

is being minimized.

For the plant in modal form, a modal weighting used for LQG design reduces to a diagonal matrix $\mathbf{Q}$. When the weighting is applied to the plant a priori, it suffices to choose $\mathbf{Q}=\mathbf{I}_{[n \times n]}$ for the LQG design. The matrix $\mathbf{R}$ is chosen as $\mathbf{R}=\alpha \cdot \mathbf{I}_{[r \times r]}$ with the design parameter $\alpha>0, \alpha \in \mathbb{R}$.

3.5.2. Normalization. When comparing different actuator configurations with a fixed set of measurements, a physically motivated choice of normalization is to fix the controller $\mathscr{H}_{2}$ norm $\|\mathbf{K}\|_{2}:=k$ which can be interpreted as a fixed average energy available for control. This also requires that the produced controllers are stable and strictly proper so that their $\mathscr{H}_{2}$ norm is finite. Strictly properness holds true for a standard LQG controller, and strong stabilization can be provided by a series of adapted design approaches if necessary; see [14].

The relationship between the controller $\mathscr{H}_{2}$ norm and the design weight $\alpha$ is close to linearity in a loglog-scaling, so $\alpha$ can be found quickly (by few design iterations) by using a loglog-interpolation to obtain a target controller $\mathscr{H}_{2}$ norm. The choice of the target $\mathscr{H}_{2}$ norm of the controller can be obtained a priori by evaluating the system's singular value plot (open-loop and closed-loop), see [13, 15].

For a strictly proper, open-loop stable plant, it is useful to formulate a closed-loop performance index as the ratio of the closed-loop and open-loop transfer $\mathscr{H}_{2}$ norms

$$
\eta_{\mathrm{LQG}}=1-\frac{\left\|\mathbf{G}_{c l}\right\|_{2}}{\left\|\mathbf{G}_{o l}\right\|_{2}} \text {. }
$$

Thereby, $\eta_{\mathrm{LQG}}=1$ is perfect attenuation while $\eta_{\mathrm{LQG}}=$ 0 indicates no improvement. If the original system is not strictly proper, the nonzero D matrix is ignored for this comparison. This can be justified because the investigation aims to assess state controllability/observability which is invariant in $\mathbf{D}$.

3.6. Actuator Size Optimization. To optimize structural actuator size a model of the benefit and costs due to size variation needs to be established. One simple model is

$$
\mathbf{u}=\mathbf{u}_{\max } e,
$$

where $e$ indicates the normalized input effort that is mapped via actuator capabilities $u_{\max }$ to the actual system inputs $u$ in (5).

If the actuation effort increases linearly with their spatial length $l$, this model can be extended to

$$
\mathbf{u}=\underbrace{\widehat{\mathbf{u}}_{\max } \frac{1}{l}}_{\mathbf{u}_{\max }} e,
$$

where $\widehat{\mathbf{u}}_{\max }$ are the actuator capabilities for unit length. Likewise, other actuation effort models can be implemented.

A relative controllability-based actuator sizing index can be derived from (15) in which the relevant $j$ th actuator modal norm includes the effort model (24) or(25)

$$
\left\|\mathbf{G}_{i j}\right\|_{2} \cong \frac{\left\|\mathbf{C}_{m i}\right\|_{2}\left\|\mathbf{B}_{m i} \mathbf{u}_{\max }\right\|_{2}}{2 \sqrt{\zeta_{i} \omega_{i}}} .
$$

3.7. Actuator Bandwidth Estimation. To quantify the performance degradation due to limited actuator bandwidth, it is useful to relate the $\mathscr{H}_{2}$ norm of the control inputperformance output transfer function $\mathbf{G}_{z u}$ system actuated by a perfect actuator and one with limited bandwidth. Assuming generic PTn low-pass behaviour of the actuator modeled by a transfer function $G_{\mathrm{PT} n}$, the actuation capability can be formulated as

$$
\eta\left(\omega_{\mathrm{c}}\right)=\frac{\left\|\mathbf{G}_{z u} G_{\mathrm{PTn}}\right\|_{2}}{\left\|\mathbf{G}_{z u}\right\|_{2}} .
$$

3.8. Robustness of Positioning Optimization. In this work, the methodologies outlined above are robustified against plant perturbations through a multimodel approach. Consider a performance index $\alpha_{l}$ (such as the positioning indices or actuator capability indices developed above) related to a candidate input or output $l$. Provided that the relevant occurring plant perturbations can be captured by a set of plants $\mathcal{P}=\left\{\mathbf{P}_{i}, i=1, \ldots, n_{p}\right\}$, compute the performance index for each $\mathbf{P}_{i}$, yielding $\alpha_{l i}$. A robustified performance index is given by

$$
\tilde{\alpha}_{l}=\min _{i=1, \ldots, n_{p}} \alpha_{l i} \quad \text { or } \quad \tilde{\alpha}_{l}=\frac{1}{n_{p}} \sum_{i=1}^{n_{p}} \alpha_{l i}
$$

Remark. Note that this aggregation does not consider the plant perturbation explicitly and only contains the filtered information from the performance indices. An alternative robustification approach is shown by the authors in [15], where the variation of SISO transfer function properties (phase, magnitude) are investigated to exclude input/output combinations when the variations are too large to allow effective robust control.

Note, moreover, that the developed performance indices are formulated to also optimize for robustness against process and measurement noises: process noise acts as external plant disturbance, so all controllability- and observabilityrelated indices lead to actuator/sensor-choices which are optimal to attenuate process noise effects on the plant. Likewise, the sensor positioning indices above typically lead to sensor choices with high signal-to-noise ratio (SNR) which renders the sensor configuration well suited to handle measurement noise. Note finally that knowledge of statistical noise properties can be introduced by suitable disturbance weighting; also the LQG validation methodology [13] can directly incorporate noise characteristics. 


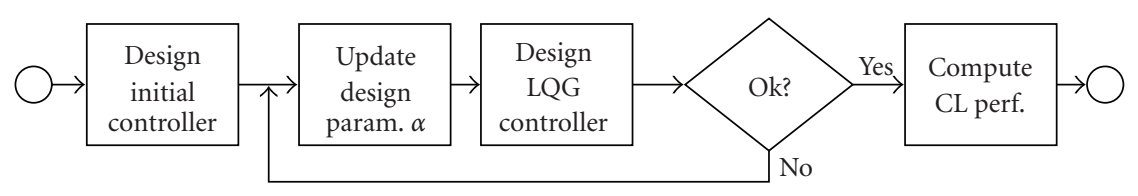

FIGURE 3: Flow chart of normalized LQG closed-loop validation approach.

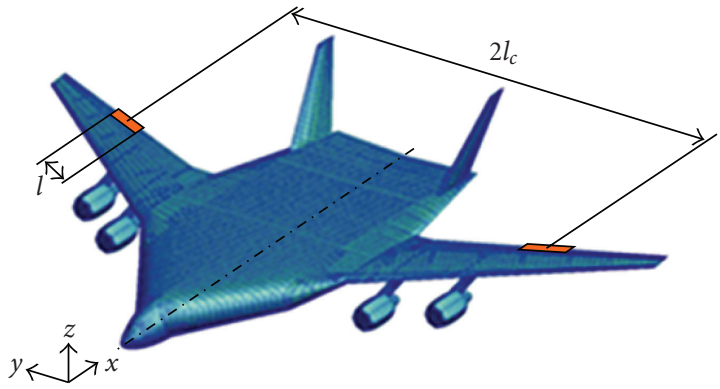

FIGURE 4: Illustration of the utilized BWB aircraft model with one modeled control surface (spanwise length $l$, center $y$-coordinate $l_{c}$ ).

\section{Aircraft Model}

The proposed placement methods are applied to a large BWB passenger aircraft. The aircraft structural mechanics were available from an FE model (see Figure 4). Aerodynamic data and the rigid-body motion were in development at the time of this study. Hence, the input/output placement targeted the structural modes' controllability and observability.

4.1. Structural Model. The structural model of the BWB aircraft model is detailed and utilized in [16]. Related, later work in BWB modeling is demonstrated in $[17,18]$. Two relevant parameters - fuel and payload mass — are considered. In this study, four mass cases are considered that exhibit characteristic behavior: M0 (no fuel, no payload), M1, M2 (intermediate cases), and M3 (max. fuel, max. payload).

The model is transformed to general continuous-time state-space form (A, B, C, D) and reduced to 60 elastic states (30 flexible modes). The boundary conditions of these models are free, however; the rigid-body modes are removed from the model for the present task.

The considered 196 inputs are comprised of:

(i) 49 input locations on each wing, 2 signals each: vertical force $F_{z}$ and pitch moment $M_{y}$.

The considered 556 outputs are comprised of

(i) 60 measurement nodes across the structure (24 in the fuselage, 18 per wing, 6 degrees of freedom each: $x, y, z$-displacements, rotations about the $x, y, z$ axis).

(ii) 196 collocated outputs to the inputs (wing rear edges, 49 per wing, $z$-displacement and rotation about the $y$-axis (i.e., pitch axis)).

The defined parameter-varying structure-only state space models were transformed to modal form (5)-(6). To do so,

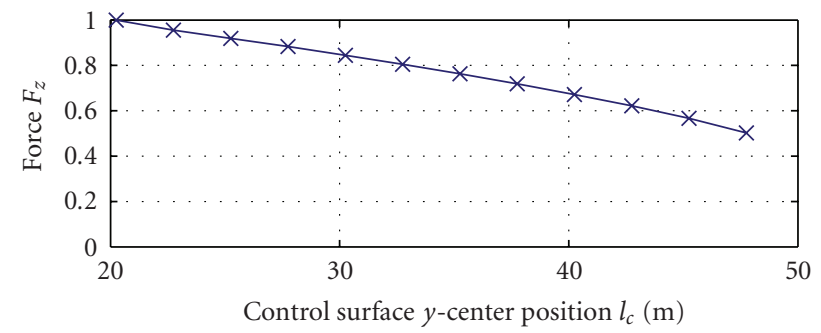

(a)

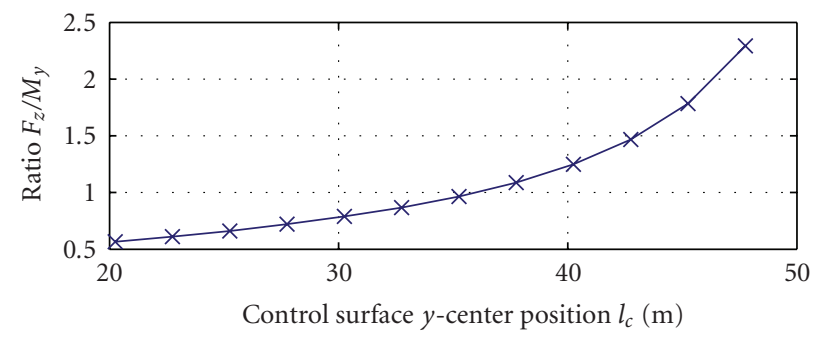

(b)

FIGURE 5: Force $F_{z}$ and ratio $F_{z} / M_{y}$ (both normalized) due to unit deflections of each $2.5 \mathrm{~m}$ control surface across the wingspan in steady flow at cruise flight conditions.

the $\mathbf{D}$ matrices (present from prior model reduction and DC gain matching) are discarded and set to 0 which is acceptable for the positioning optimization since only the dynamics of the retained vibration modes are of importance. The correct gain distribution between the matrices $\mathbf{B}$ and $\mathbf{C}$ is obtained by using the collocated inputs and outputs.

4.2. Control Surface Model. A basic control surface model is defined to relate the nodal force and moment inputs and to account for the control surface effectiveness that varies along the wingspan. Geometric and simulation data originating from a quasistatic aeroelastic trim analysis of a roll manoeuvre were used. For each wing, 12 trailing edge control surfaces of $l=2.5 \mathrm{~m}$ spanwise length were modeled. The steady-flow forces and moments for a fixed deflection of each of these surfaces were computed and reduced to their hinge center points (see Figure 5). This analysis was carried out at cruise altitude and speed $\left(M a=0.85\right.$ and $p_{0}=$ $14.4^{k N} / m^{2}$, corresponding to a cruise altitude of $35000 \mathrm{ft}$ ).

Assuming uniform line support of the control surface on the wing trailing edge, consistent node loads were computed. Using them as weighting for each involved node for a given control surface position (and spanwise length), a control surface input vector $\mathbf{b}_{\text {in }}$ can be modeled by weighted summation. 
4.3. Excitation and Performance Criteria Weightings. In order to optimize the actuator and sensor positions for the given control tasks, system excitation as well as performance formulations have to be considered.

The main system excitation is assumed to be wind turbulence (modeled by a 1D von Karman turbulence filter as defined in [19]) applied to the vertical force inputs $F_{z}$ along the wings' rear edges.

Two performance criteria are defined.

(i) Load performance criterion. An amplitude-based criterion is used as load-proportional quantity. The vector norm over all vertical deflections $s_{k, q z}$ of the wing sensor nodes $k \in S_{\text {wing }}$ is used

$$
z_{\text {load }}=\left\|\left[\begin{array}{c}
s_{1, q z} \\
\vdots \\
s_{k, q z} \\
\vdots
\end{array}\right]\right\|
$$

(ii) Comfort performance criterion. The fuselage sensor positions $k \in S_{\text {fuselage }}$ are chosen for comfort evaluation. Their accelerations in the $x$-, $y$-, and $z$-directions $\left(s_{k, a x}, s_{k, a y}, s_{k, a z}\right)$ are weighted using the respective ISO 2631 2631-filters [20] (yielding $\left.s_{k, a x, \text { ISO }} s_{k, a y, \text { ISO }} s_{k, a z, \text { ISO }}\right)$; the vector norm of these weighted signals is taken as measure of average ride comfort

$$
z_{\mathrm{comf}}=\left\|\left[\begin{array}{c}
\vdots \\
s_{k, a x, \mathrm{ISO}} \\
\vdots \\
s_{k, a y, \mathrm{ISO}} \\
\vdots \\
s_{k, a z, \mathrm{ISO}} \\
\vdots
\end{array}\right]\right\|
$$

\section{Results}

This section reports the results obtained by applying the described methods to the described BWB aircraft system model. The results are interpreted while taking into account the posed modeling assumptions. Finally, an actuator and sensor baseline concept is proposed for the BWB aircraft.

5.1. Actuator Positioning Optimization. For the actuator positioning task, the positioning indices $\sigma_{\text {act }}$ (see (15)), $\pi_{\text {act }}$ (see (19)) and the LQG closed-loop validation index $\eta_{\text {LQG }}$ (see (23)) are evaluated for each candidate actuator. The candidate actuators are modeled with fixed length $l=2.5 \mathrm{~m}$ and varying center position $l_{c}$ by interpolation of the data from Section 4.2. The system performance formulations for load

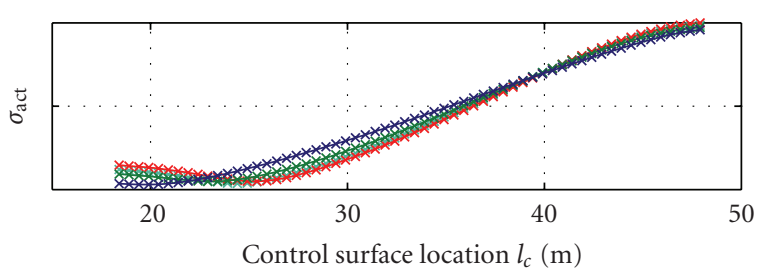

(a)

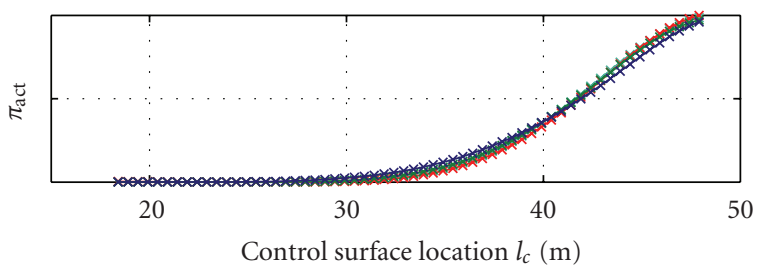

(b)

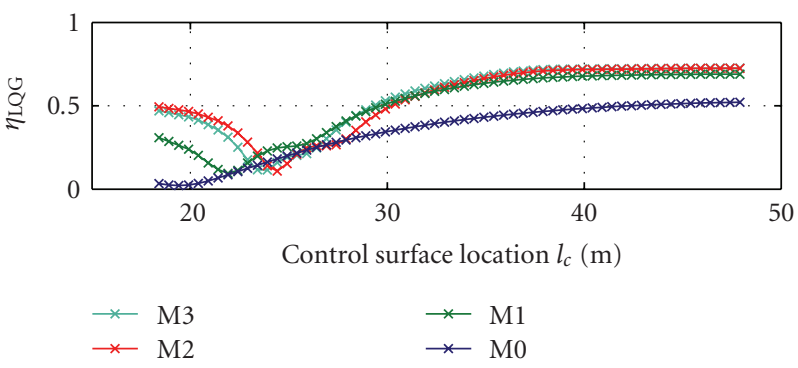

(c)

FIGURE 6: Actuator positioning performance indices for load performance: Gawronski $\sigma_{\text {act }}$ from (15) [2], combined index $\pi_{\text {act }}$ from (19) [7], and LQG index $\eta_{\mathrm{LQG}}$ from (7).

(29) and comfort (30) are implemented by modewise input and output weighting capturing the excitation spectrum and the performance output formulations, respectively.

The evaluation results are plotted in Figure 6 for the load criterion and in Figure 7 for the comfort criterion.

(i) The best overall positions are always at the outmost wing locations.

(ii) For the load criterion, only the first few modes do have significant influence, whereas the comfort criterion involves more relevant modes.

(iii) The different fuel/payload cases differ slightly, but all show the same fundamental statement.

(iv) $\eta_{\text {LQG }}$ has the same global optimum, however, a larger range of near-optimal positions is indicated.

5.2. Sensor Positioning Optimization. For the sensor positioning task, the positioning indices $\sigma_{\text {sens }}$ (see (17)) and $\pi_{\text {sens }}($ see $(20)$ ) are evaluated for each candidate sensor for both performance formulations (load criterion (29) and comfort criterion (30)). The applied system weightings are comprised of a modewise input weighting to capture the excitation spectrum and a modewise output weighting that reflects the modal contributions in the performance output formulations. Additionally, a set of sensors with low modegain correlation across all mass cases are found through 


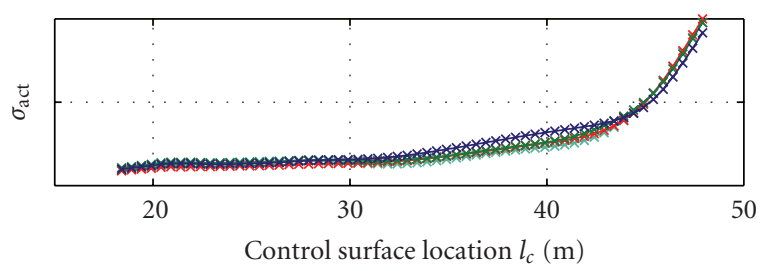

(a)

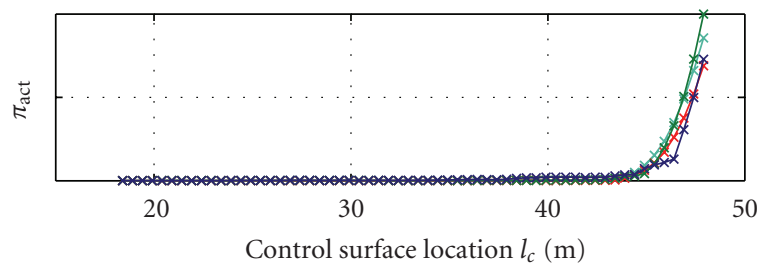

(b)

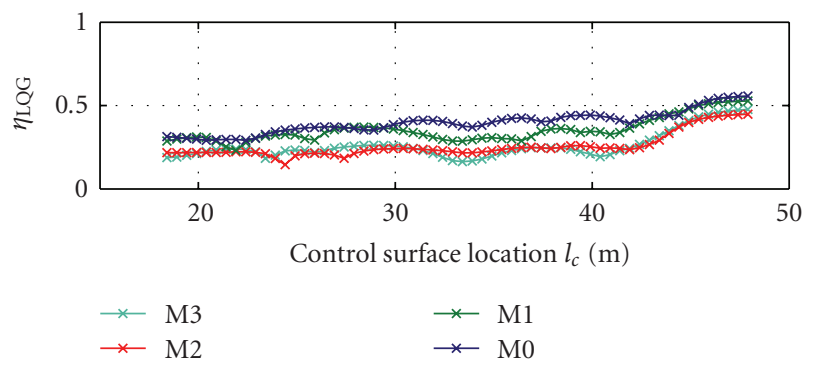

(c)

FIgURE 7: Actuator positioning performance indices for comfort performance: Gawronski $\sigma_{\text {act }}$ from (15) [2], combined index $\pi_{\text {act }}$ from (19) [7], and LQG index $\eta_{\text {LQG }}$ from (23).

the sensor positioning algorithm in Section 3.4. The sensor positioning indices are depicted in Figure 8 for the load criterion and in Figure 9 for the comfort criterion. Figure 10 shows the sensor positioning proposal obtained by the sensor positioning algorithm in Section 3.4 for a chosen maximum admissible correlation of $r_{\max }=0.5$.

(i) The best sensor positions are indicated by both positioning criteria at the wingtips, for both performance formulations (load and comfort).

(ii) The next best sensor positions which are sufficiently uncorrelated are located near the wing roots and in the fuselage, however with significantly lower performance criterion values (compare Figures 8 and 9).

(iii) The sensors encircled in Figure 10 are chosen as primary feedback sensor candidates.

5.3. Estimation of Optimal Control Surface Length. The effect of varying control surface length is evaluated via the method proposed in Section 3.6 for both performance criteria (load (29) and comfort (30) criteria). A pair of control surfaces of varying length $l=0.5 \cdots 30 \mathrm{~m}$ at the outmost wing positions is modeled. System weightings are applied as in Section 5.1 and the derived indices $\sigma_{\text {act }}^{*}$ (from (15) and (26)

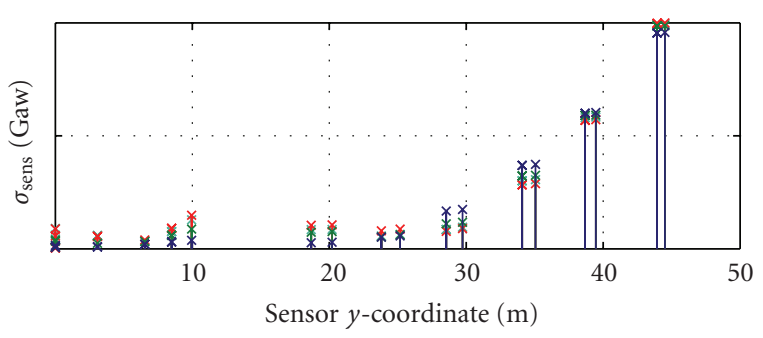

(a)

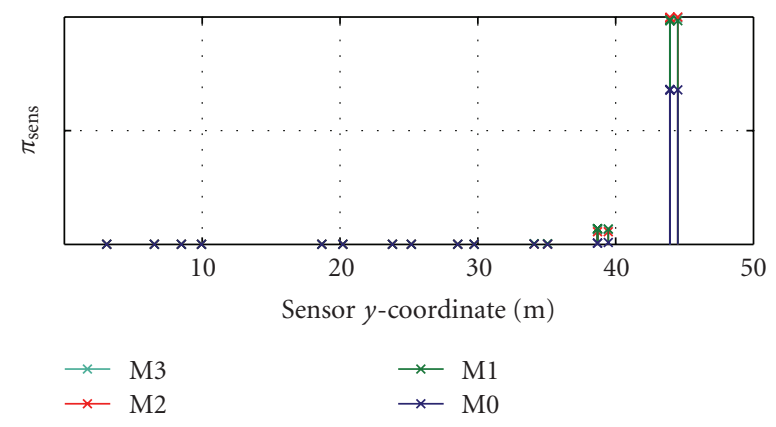

(b)

FIGURE 8: Sensor positioning performance indices for load performance: Gawronski $\sigma_{\text {sens }}$ from (17) [2], combined index $\pi_{\text {sens }}$ from (20) [7].

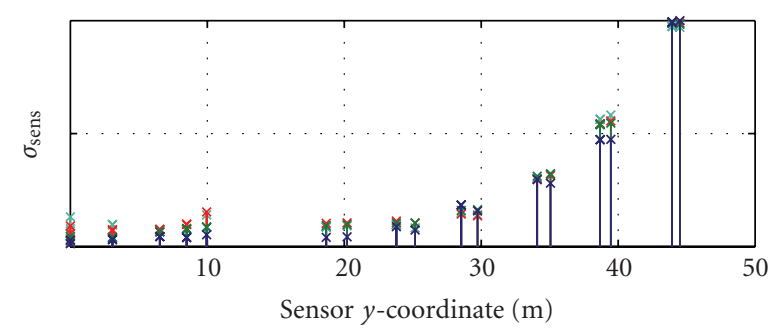

(a)

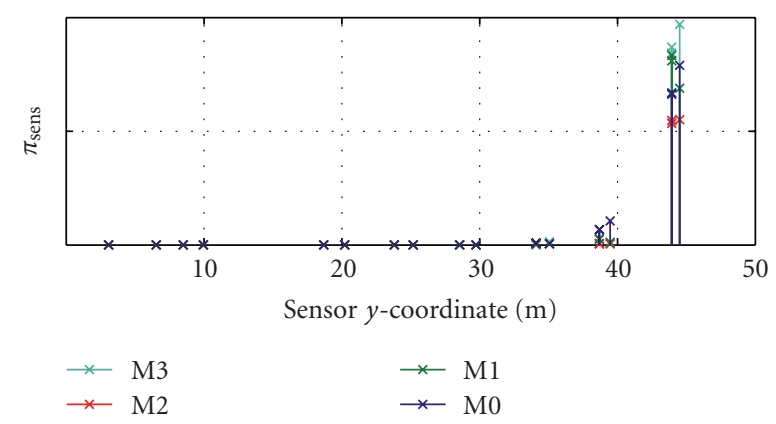

(b)

FIGURE 9: Sensor positioning performance indices for comfort performance: Gawronski $\sigma_{\text {sens }}$ from (17) [2], combined index $\pi_{\text {sens }}$ from (20) [7].

with effort model (24)) and $\eta_{\mathrm{LQG}}^{*}$ (from (23) with effort model (24)) are evaluated.

The sizing results for the load criterion are shown in Figure 11 and those for the comfort criterion are shown in Figure 12. A threshold of $80 \%$ of the maximal value is indicated therein to help in the result interpretation: 


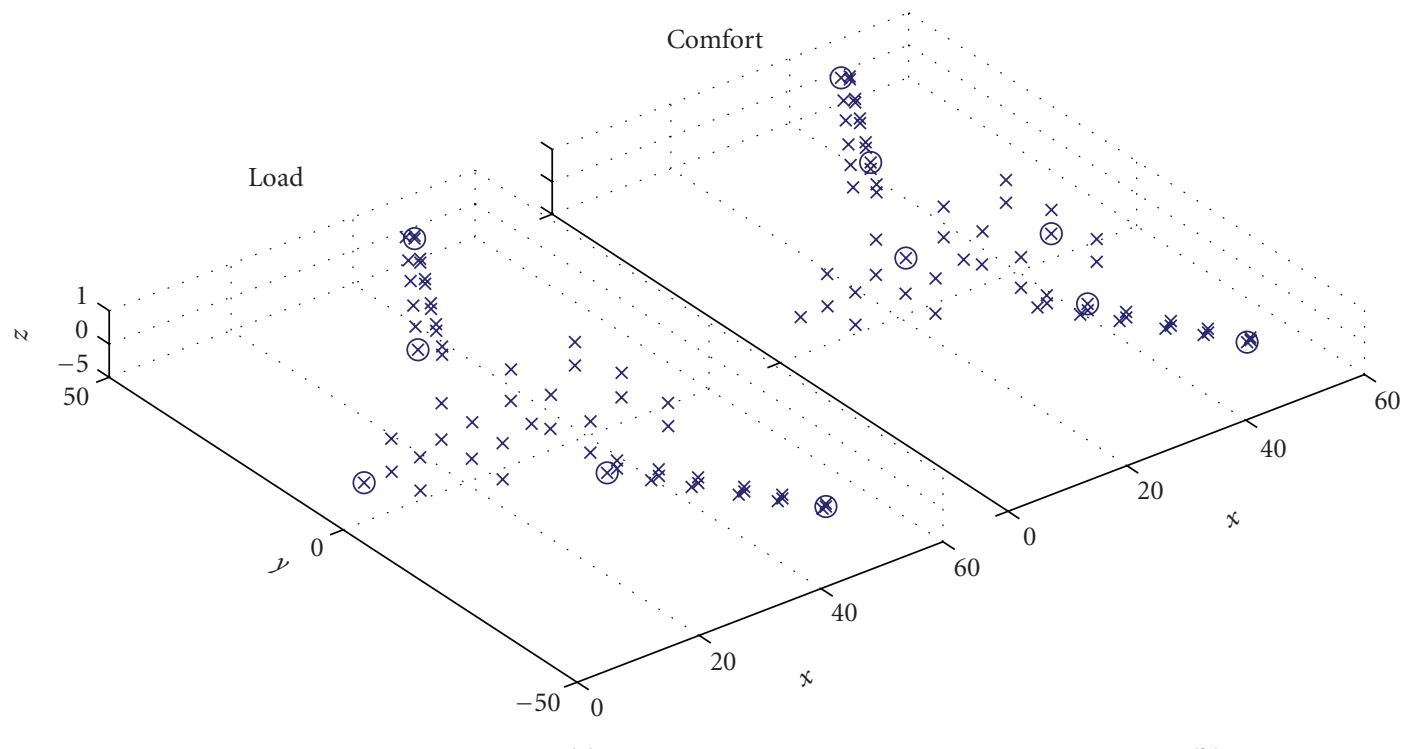

(a)

(b)

FigURE 10: Sensor placement results, min. correl. distance $r_{\max }=0.5$ ((a) load criterion, (b) comfort criterion).

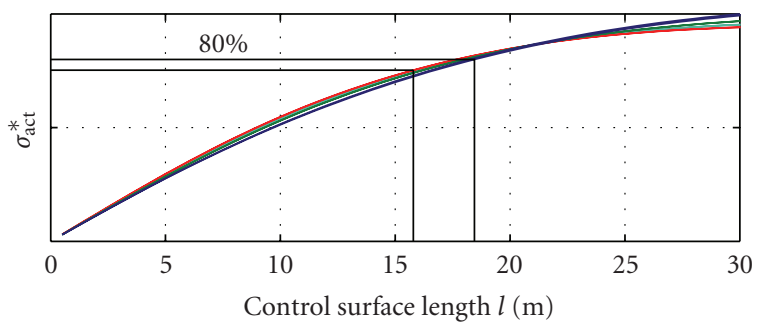

(a)

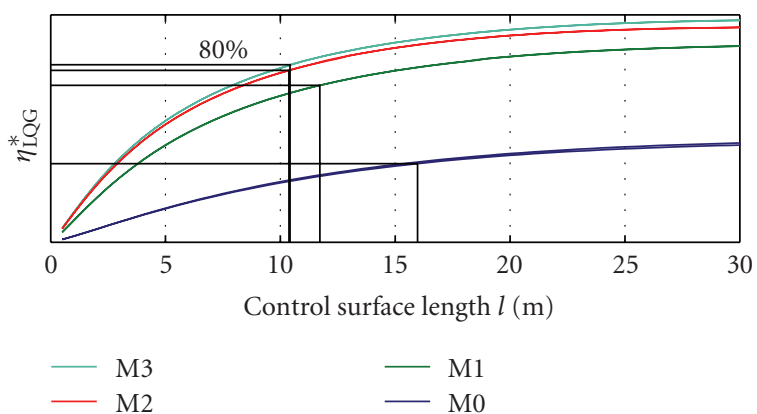

(b)

Figure 11: Control surface length influence on load: open-loop criterion $\sigma_{\text {act }}^{*}$, LQG validation $\eta_{\mathrm{LQG}}^{*}$.

nonmodeled constructive and cost constraints render small control surface lengths favorable, so it is of interest to find the smallest actuator length that achieves a desired performance level.

(i) For the load criterion, the indicated smallest effective actuator length lies between 10 and $20 \mathrm{~m}$ according to both the energy and the LQG closed-loop criteria.

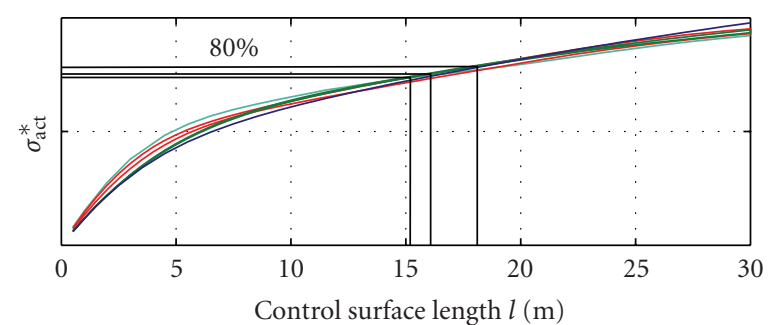

(a)

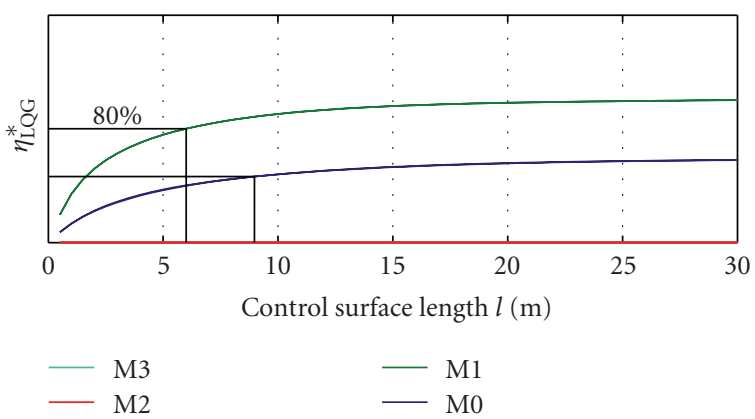

(b)

FIGURE 12: Control surface length influence on comfort: open-loop criterion $\sigma_{\text {act }}^{*}$ LQG validation $\eta_{\mathrm{LQG}}^{*}$.

(ii) For the comfort criterion, the indicated smallest effective actuator length lies between 15 and $20 \mathrm{~m}$ according to the energy criterion $\sigma_{\text {act }}^{*}$, while the closed-loop validation indicates a smaller length between 5 and $10 \mathrm{~m}$ as effective.

(iii) The energy-related criterion is strongly dependent on the formulation of input signal energy. The presented investigations were carried out with the 


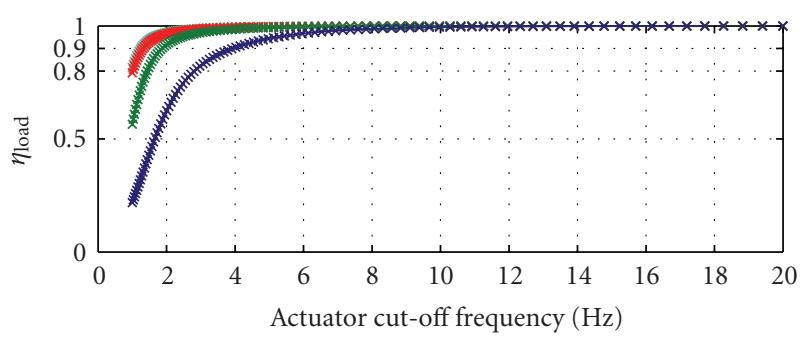

(a)

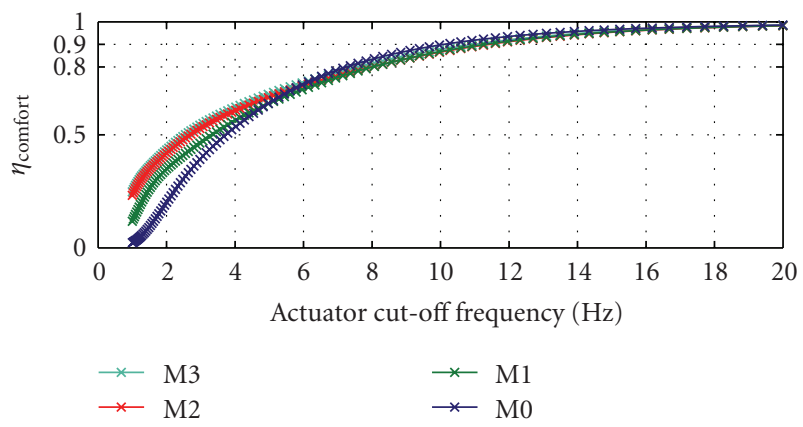

(b)

Figure 13: Actuator bandwidth estimation ((a) load criterion, (b) comfort criterion).

angular control surface deflection as input signal (see Section 3.6 for other options).

(iv) The obtained vibration reduction strongly depends on the mass case.

The following limitations and considerations have to be kept in mind.

(i) The utilized effort model (24) considers equal deflections of small and large control surfaces equally in terms of effort. The study can be adapted to incorporate more complex effort models.

(ii) Experimental data is available for control surfaces of $2.5 \mathrm{~m}$ length. Extrapolation far beyond this value (as here, up to $30 \mathrm{~m}$ ) introduces uncertainty.

5.4. Estimation of Actuator Bandwidth Requirements. The actuator bandwidth-performance tradeoff is evaluated for a pair of control surfaces of length $l=2.5 \mathrm{~m}$ at the outmost wing positions according to Section 3.7 for both performance formulations (load (29) and comfort (30)). The actuator dynamics is modeled as a generic 2nd-order lowpass $G_{\mathrm{PT} 2}=\omega_{c} /\left(s^{2}+2 \zeta \omega_{c} s+\omega_{c}^{2}\right)$ with cut-off frequency $\omega_{c}$ and $\zeta=1$. This study is carried out on the strictly proper part of the weighted systems ( $\mathbf{D}$ is set to $\mathbf{0}$ ).

The results in Figure 13 show that the bandwidth requirements for control surfaces to obtain $80 \%$ to $90 \%$ of the open-loop static actuation gain to performance outputs is about $4 \mathrm{~Hz}$ for the load criterion and 9 to $12 \mathrm{~Hz}$ for the comfort criterion. It is evident that the load criterion poses significantly lower bandwidth requirements than the comfort criterion does. The empty aircraft requires highest actuation bandwidth in terms of the load criterion. These differences are not pronounced for the comfort criterion where a number of modes up to $10 \mathrm{~Hz}$ are equally important.

5.5. Remarks on the Robustness of the Results. This study considers a set of plants at relevant parameter cases (selected a priori). The obtained results vary with the underlying plants, and their approximate sensitivities to plant parameter changes thus become available and yield important information for subsequent control designs.

\section{Conclusions}

In this work, methods for parameter studies and optimization of actuator and sensor positions were developed and tested. Results were computed for the BWB aircraft configuration, and specifications of an actuator and a feedback sensor baseline concept addressing positioning, actuator sizing, and bandwidth estimation were established.

The first part of the report collects the theoretical basics for the actuator and sensor positioning optimization task. The modal representation of structure state space models together with transformations and extensions is presented.

Based on this system model structure, actuator and sensor positioning methods are developed together with additional methods for actuator size and bandwidth estimations and closed-loop validation algorithms.

The used system model is built up from the structureonly state space model of a large BWB passenger aircraft in different mass cases with suitably defined inputs and outputs. By augmenting this model with existing design information, such as turbulence excitation spectrum weightings, performance criteria formulations, and basic control surface modeling including quasistatic aeroelastic effectiveness data, the system model is completed. The developed tools are then applied to the BWB aircraft system model.

The main outcomes show that for this aircraft model the structural modes relevant for the considered loads and comfort criteria can be best actuated by wingtip actuators. The wingtips are also best suited for sensing the relevant dynamics.

Estimation of optimal control surface length for subsequent vibration control is sensitive on the underlying assumptions, and results of the criteria are less coherent. It is, however; indicated that large surfaces (10 to $20 \mathrm{~Hz}$ spanwise length) are effective for actuating the load-relevant dynamics, while short surfaces of 5 to $10 \mathrm{~Hz}$ spanwise length provide most of the achievable effect on the comfort-relevant structural modes.

Finally, the actuator bandwidth requirements have been estimated to approximately $4 \mathrm{~Hz}$ for the loads criterion and 9 to $12 \mathrm{~Hz}$ for the comfort criterion.

The proposed methods for the actuator and sensor positioning and design tasks have been demonstrated at a large BWB passenger aircraft model but can directly be applied to other flexible structure systems. The given range of different approaches allows the control engineer to compute validated quantitative results for positioning and actuator studies and to detect critical uncertainties in the posed assumptions and in the problem data. 
Related work in progress includes comfort improvement through control [16]. Control designs on the same BWB aircraft configuration that aim also at loads alleviation and flight control have been carried out in [21, 22]. In [23], an LPV control design is performed and convex control design approaches are in the process of submission.

For a redesigned BWB aircraft structure, the actuator/sensor positioning task was repeated, and alternative criteria and methodologies have been proposed in [13] that complement the methods proposed here. Future work include lateral and longitudinal scheduled control law designs on the redesigned aircraft configuration with simultaneous handling qualities, loads alleviation, and comfort improvement goals.

\section{Acknowledgments}

This work was financially supported by the European Union Framework Programme 7 under the FP7 Project no. 213321 [1]. The authors are grateful to Dr. Klimmek of the DLR to provide them with aeroelastic trim analysis data for input weighting.

\section{References}

[1] ACFA 2020 Consortium, "Active control of flexible 2020 aircraft (ACFA 2020), EU FP7 project no. 213321,” January 2011, http://www.acfa2020.eu.

[2] W. Gawronski, Advanced Structural Dynamics and Active Control of Structures, Springer, New York, NY, USA, 2004.

[3] S. Skogestad and I. Postlethwaite, Multivariable Feedback Control, John Wiley \& Sons, New York, NY, USA, 1996.

[4] M. Van De Wal and B. De Jager, "Review of methods for input/output selection," Automatica, vol. 37, no. 4, pp. 487$510,2001$.

[5] A. Hać, "Distribution of actuators in vibration control of adaptive structures," in Proceedings of the American Control Conference, pp. 4295-4299, Seattle, Wash, USA, June 1995.

[6] S. Leleu, H. Abou-Kandil, and Y. Bonnassieux, "Piezoelectric actuators and sensors location for active control of flexible structures," IEEE Transactions on Instrumentation and Measurement, vol. 50, no. 6, pp. 1577-1582, 2001.

[7] C. Westermayer, A. Schirrer, M. Hemedi, and M. Kozek, "An advanced criterion for optimal actuator and sensor placement on complex flexible structures," in Proceedings of the IFAC Workshop on Control Applications of Optimisation, Jyväskylä, Finland, 2009.

[8] C. Benatzky, Theoretical and experimental investigation of an active vibration damping concept for metro vehicles, dissertation, Vienna University of Technology, Vienna, Austria, October 2006.

[9] A. Schirrer, Co-simulation of rail car body vibration control with SimPACK, M.S. thesis, Vienna University of Technology, 2007.

[10] F. Fahroo and Y. Wang, "Optimal location of piezoceramic actuators for vibration suppression of a flexible structure," in Proceedings of the 36th Conference on Decision and Control, pp. 1966-1971, San Diego, Calif, USA, December 1997.

[11] F. Wu, Control of Linear Parameter Varying System, Ph.D. thesis, Department of Mechanical Engineering, University of California at Berkeley, Berkeley, Calif, USA, 1995.

[12] A. Hac and L. Liu, "Sensor and actuator location in motion control of flexible structures," Journal of Sound and Vibration, vol. 167, no. 2, pp. 239-261, 1993.
[13] M. Hemedi, A. Schirrer, C. Westermayer, and M. Kozek, "Performance evaluation of an input/output selection criterion via normalized LQG closed-loop comparison," in Proceedings of the 17th IEEE Mediterranean Conference on Control \& Automation, Thessaloniki, Greece, June 2009.

[14] M. Vidyasagar, Control System Synthesis: A Coprime Factorization Approach, MIT Press, Cambridge, Mass, USA, 1988.

[15] M. Hemedi, A. Schirrer, C. Westermayer, and M. Kozek, "Integrated input-output selection strategy for robust control of complex parameter varying systems," in Proceedings of the 10th International Conference on Motion and Vibration Control (MOVIC '10), Japan Society of Mechanical Engineers, Tokyo, Japan, August 2010, no. 10-203.

[16] P. Zuziak, Z. Sika, M. Valasek, T. Vampola, and T. Klimmek, "Vibration control of flexible aircraft with respect to passengers comfort," in Proceedings of the ISMA International Conference on Noise and Vibration Engineering, 2010.

[17] F. Stroscher, O. Petersson, and M. Leitner, "Aircraft structural optimization subject to flight loads-application to a wide body commercial aircraft configuration," in Proceedings of the Workshop on Aerostructures of the European Aeronautics Science Network (EASN'10), 2010.

[18] F. Stroscher, O. Petersson, and M. Leitner, "Structural optimization framework for aircraft subject to transient maneuver and gust loads," in Proceedings of the 13th AIAA/ISSMO Multidisciplinary Analysis Optimization Conference, 2010.

[19] F. Hoblit, Gust Loads on Aircraft: Concepts and Applications, American Institute of Aeronautics and Astronautics, New York, NY, USA, 1988.

[20] ISO, "ISO2631-1: Mechanical vibration and shock - evaluation of human exposure to whole-body vibration. Part 1: General requirements," International Organization for Standardization, May 1997, Corrected and reprinted July 2007.

[21] A. Schirrer, C. Westermayer, M. Hemedi, and M. Kozek, "A comprehensive robust control design and optimization methodology for complex flexible-structure systems," in Proceedings of the 18th Mediterranean Conference on Control and Automation (MED '10), pp. 1037-1042, Marrakesh Morocco, June 2010.

[22] A. Schirrer, C. Westermayer, M. Hemedi, and M. Kozek, "LQbased design of the inner loop lateral control for a large flexible BWBtype aircraft," in Proceedings of the IEEE International Conference on Control Applications, Tokyo, Japan, September 2010.

[23] C. Westermayer, A. Schirrer, M. Hemedi, and M. Kozek, "Linear parameter-varying control of a large blended wing body flexible aircraft," in Proceedings of the 18th IFAC Symposium on Automatic Control in Aerospace, Nara, Japan, September 2010. 

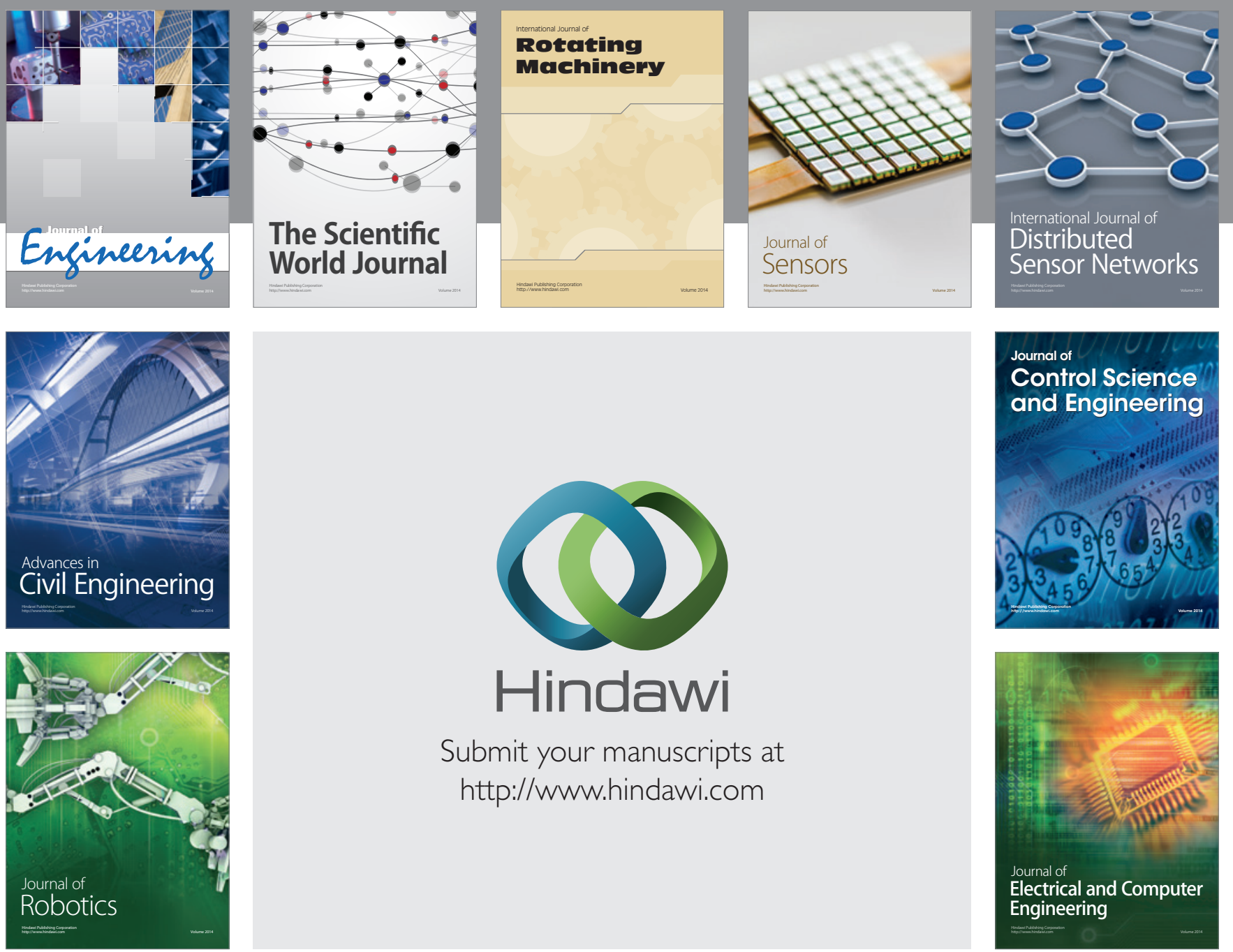

Submit your manuscripts at

http://www.hindawi.com
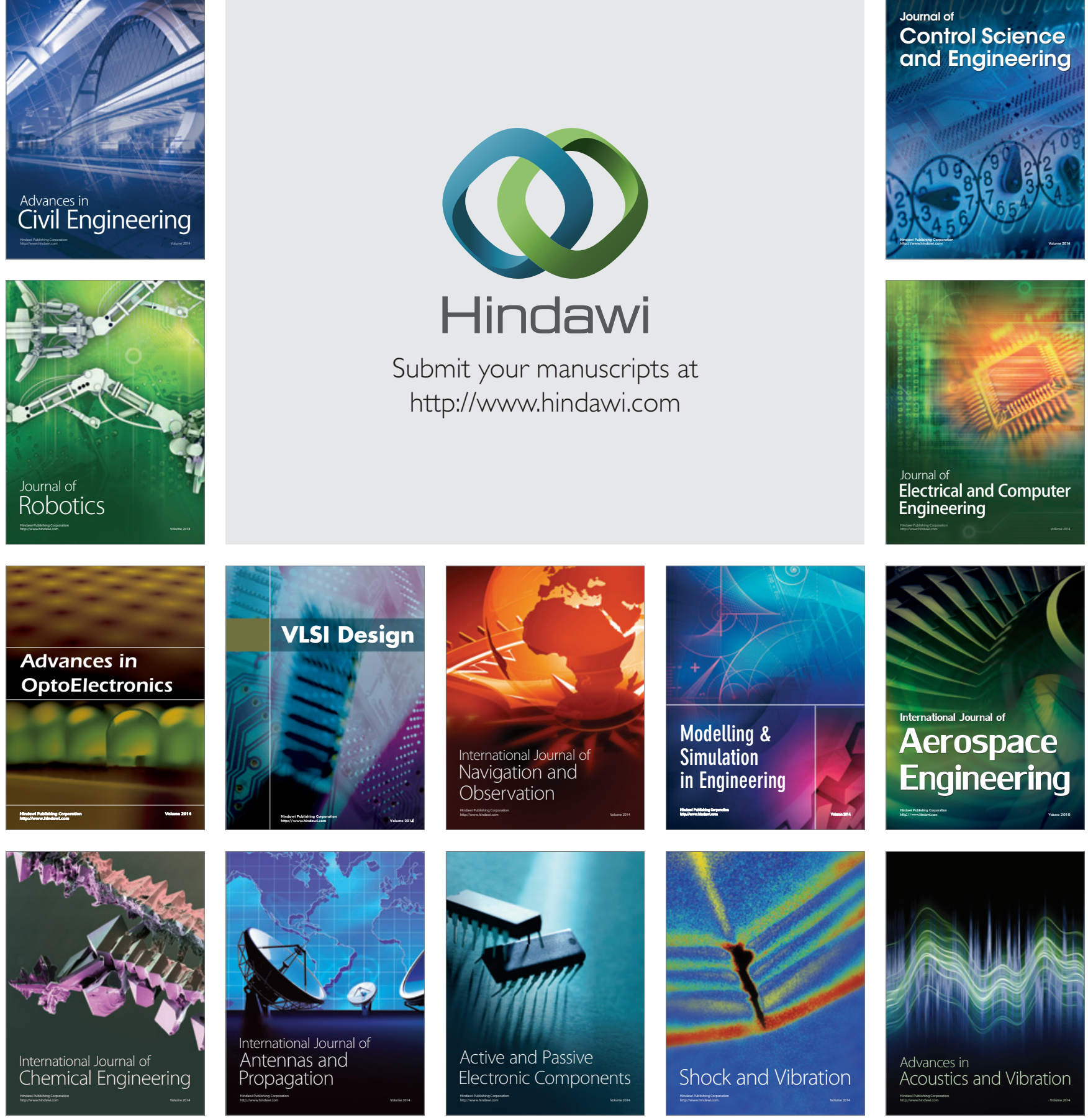